\title{
A DEEP LEARNING BASED TRAFFIC CLASSIFICATION IN SOFTWARE DEFINED NETWORKING
}

\author{
Rahma Hammedi \\ Prince Lab, ISITCOM, University of Sousse, Sousse, Tunisia
}

\begin{abstract}
Moving from legacy internet applications, such as web, and e-mail, to dynamic complex applications, such as video streaming, and file sharing, improves the underlying network architectures to provide new services with high QoS requirements. Thus, a new network paradigm is developed and deployed; Software Defined Networking (SDN). As a result of the significant impact of using the internet, network traffic is growing up, and the network itself is becoming more overloaded. Therefore, new tools that can cover new requirements for high-quality services are becoming mandatory. Inherently, traffic classification (TC) has gained continuous interest as an important decision- centric approach to deliver the quality of service. The current main challenge is how to classify flows efficiently. In this work, a new method of classification for incoming flows is proposed. It is based on Deep learning and consists of using the MultiLayer Perceptron Model (MLP) to classify flows according to its constraints throughput and delay. Experimental results prove that the proposed approach outperforms parallel solutions in terms of precision. It can classify traffic with more than $97.7 \%$ precision compared to the Linear Regression classification and the Fuzzy Decision- tree model.
\end{abstract}

\section{KEYWORDS}

SDN, Traffic Classification, Deep Learning, Artificial Neural Network

\section{INTRODUCTION}

Current technological advancements in the field of networking do not cease evolving. Emerging applications require high demands on Quality of Service (QoS) such as delay and bandwidth to satisfy users. Thus, it is necessary to introduce new QoS- delivery approaches to meet the current application requirements. The current internet architectures provide only best-effort services, which cause traffic management problems. To solve this problem, it was thought to optimize the traffic classification as it is essential for the network functionality (Nguyen \& Armitage, 2008). A promising solution to address the complexity of traffic classification is SDN. SDN is a novel architecture that offers centralized management of the network (routing, classification, monitoring.) through programmable interfaces. This may ensure flexibility and fine-grained flow control. Unfortunately, current classification techniques are not evaluative. This current work aims to overcome the lack of sufficient support for performing tasks related to TC (Da Silva et al., 2015). Thus, we create a new classification method to solve these problems and to guarantee QoS. The traffic classification model is formulated to optimize traffic scheduling according to the appropriate class. The rest of this paper is structured as follows. Section 2 presents an overview of the current public architecture and techniques. In Section 3, we detail the characteristics of our proposed architecture. Finally, discussion on results and performance evaluation are presented in Section 4.

\section{RELATED WORK}

Traffic classification (TC) is defined as linking and identifying flows to traffic classes (Ng et al., 2015). For two decades, it is becoming a fundamental network function to a wide range of applications (Rezaei \& Liu, 2019) and has attracted increasing research efforts. To address this network function, some work, such as the differentiated service model (DiffServ) (Chan et al, 2006), core on early-stage traffic identification, i.e., techniques that use the information contained in the Differentiated Service Code Point (DSCP) byte in 
each packet which is associated to a Per-Hop Behavior (PHB). According to network management problems, three types of PHB have been defined:

-BEST EFFORT (BE): The network provides no quality of service. It does neither guarantee the transmission delay nor the required resource allocation.

-ASSURED FORWARDING (AF): This PHB is intended for bandwidth-sensitive flows, allowing transmitting real-time data which have the constraint of resource allocation.

-EXPEDITED FORWARDING (EF): Designed for traffic that requires strict delay. EF can also be used to build a service that requires very low latency, jitter, and packet loss.

As shown in (Burgstahle et al, 2003), defining the code (DSCP) in each incoming packet is helpful, but it suffers from scalability problems. Authors in (Karagiannis et al, 2004) proposed an approach that is based on logical port numbers. It depends on investigating the standard ports used by well- known applications. However, novel applications either use well-known port numbers to disguise their traffic or do not use standard registered port numbers. (Li \& Li J., 2014) Present traffic classifiers which dependent on Deep Packet Inspection (DPI). They focus on finding patterns or keywords in packets. These methods are only relevant to unencrypted traffic and have a lofty computational overhead. As a result, a new method is based on flow statistics. Usually, these methods employ classical machine learning (ML) algorithms (Sen et al., 2004). The effectiveness and accuracy of port-based classification, DPI, and classical machine learning methods have been declined due to the increasing variety and complexity of recent Internet applications. Recent research on network TC focuses on deep learning (Da Silva et al., 2015). Deep learning obviates the need to select features by a domain expert since it automatically selects features through training. Thus, deep learning has a considerably higher capacity of learning and efficient approach (Karagiannis et al, 2004), (Nandy, 2013) for TC. Authors in (Sandhya et al., 2017) show that SDN is a promising architecture to build efficient TC model due to its network-wide visibility (Hayes \& $\mathrm{Ng}, 2018$ ). The current SDN controller can enhance TC in large-scale networks (Morzhov \& Nikitinskiy, 2018).

SDN can face the legacy IP architecture's limitations by decoupling the control plane from the forwarding plane and moving it to a centralized node, called the SDN controller. Network devices become simply Forwarding Elements (FE) (Pérez et al., 2019).The application of deep learning in SDN networks to classify flows is divided into supervised learning and unsupervised learning. In Unsupervised Learning or clustering, no information is required on the class label of the data or the number of classes; the goal is to discover similar instances in the data. The purpose of supervised classification is to learn using training set a classification procedure, allowing classifying new instances into predefined classes. Supervised learning has two main stages:

- Training: the learning phase analyses the data provided (training dataset) and forms a classification model.

-Validation/testing: the model created during the training stage is used to classify the new illustrations. The performance of the classification system is assessed during this step.

The literature (Nandy, 2013), (Sandhya et al., 2017) determines that supervised classification outperformed unsupervised clustering. A comparison between the hierarchical classification and a battery of different supervised classification algorithms (Nandy, 2013) reveals that the hierarchical classification approach is unable to obtain a precision as precise as the supervised classification. Consequently, supervised classification is more efficient than hierarchical classification.

\section{PROPOSED ARCHITECTURE}

In this section, the proposed classification approach is described, and phases of this approach are detailed. Finally, an illustrative example of the routing application of this model is presented.

\subsection{Overall Structure}

Our approach consists on classifying the incoming flows according to two features, Delay and Throughput, into three classes defined by the DiffServ approach (ref. section 2). The selection of these features is imposed because the delay and the throughput are considered the most relevant to define the constraints required by a flow to guarantee the application routing's optimal quality. Thus, flows are classified: AF for applications with bandwidth constraints and implicitly bit rate, EF for applications with delay constraints, and BE for applications without constraints. The proposed approach is based on two phases, a phase of preparation of the dataset (feature extraction) and a flows classification phase. This process is depicted in the figure 1. 


\subsubsection{Phase1: Feature Extraction}

The extraction model receives a set of data as a pcap file obtained by capturing packets with the Wireshark tool (Kogan et al., 2013), invisibly in "promiscuous" mode, without jeopardizing data routing.

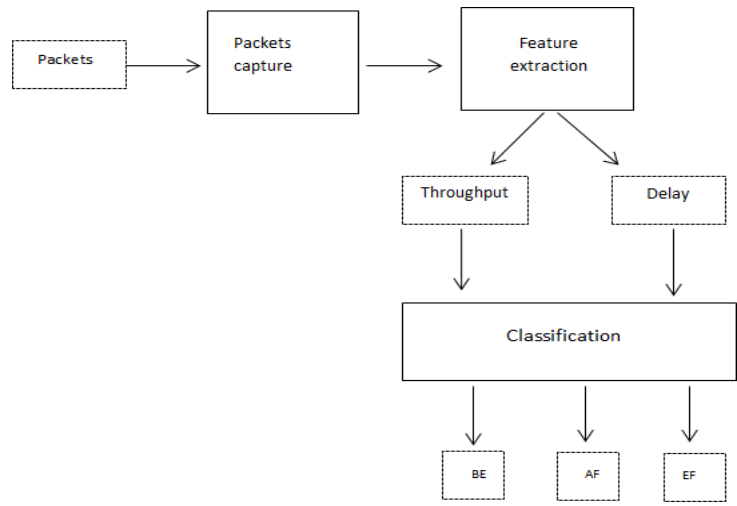

Figure 1. Process of the proposed classification model

Then, captured packets are decoded to find the required information, delay, and throughput of each flow.

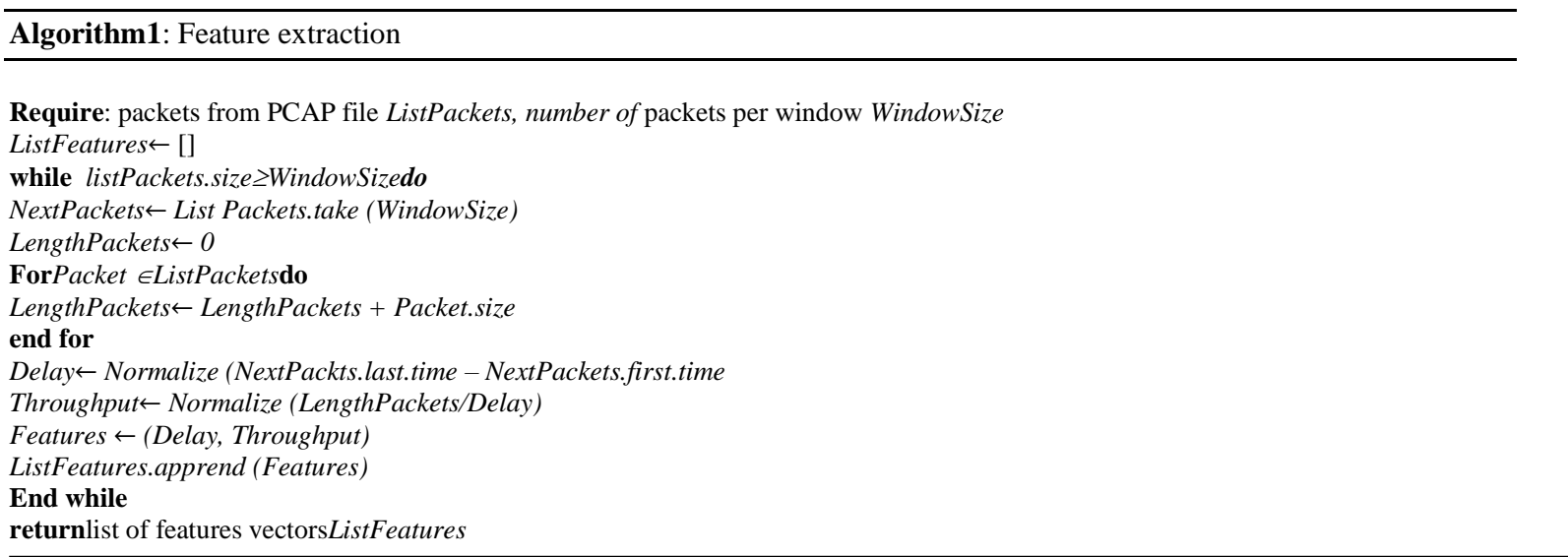

\subsubsection{Classification}

Increasing learning algorithm parameters is related to several factors, including the number of hidden layers, the activation function, choice of necessary tests, and algorithms learning. The proposed model is based on the Multilayer Perceptron (MLP) algorithm.

It is recognized as the most used model due to its highest overall accuracy (Yan et al., 2015). A neuron acquires a weighted sum of its inputs and passes it over an activation function to create an output. In this algorithm, the input vector represented the throughput and the delay values, referred to as. At the output, the three PHBs defined in the DiffServ architecture are adopted so that each flow must be classified at BE, EF, or AF class.

B: Bias

Inputs: $\mathrm{X}=(\mathrm{x} 1, \mathrm{x} 2)$

Outputs: $Y=(y 1, y 2, y 3)$

$w_{i}^{j}$ : The weight of link coupling the node $i$ from the previous layer to the node $j$ from the next layer

F: The Sigmoid Activation function is used as follows:

$$
\mathrm{f}_{\text {Sig }}(\mathrm{x})=\frac{1}{1+\mathrm{e}^{-\mathrm{x}}}
$$


The sum of the weights pre-activation is defined by the function $\mathrm{Z}(\mathrm{X})$ as follows:

$$
\mathrm{Z}(\mathrm{x} 1, \mathrm{x} 2)=\mathrm{w}_{1}^{1} \mathrm{x}_{1}+\mathrm{w}_{2}^{1} \mathrm{x}_{2}+\mathrm{B}
$$

In the second stage, we use an aggregation function whose association weights calculated in the previous phase are combined. $\mathrm{N}$ refers to the number of nodes in the neural network.

$$
\mathrm{Z}(\mathrm{X})=\sum_{1}^{\mathrm{N}} \mathrm{w}_{\mathrm{i}}^{\mathrm{j}} \mathrm{x}_{\mathrm{i}} \quad \mathrm{i}=1 \ldots \mathrm{N}
$$

In the last step the function $\mathrm{Z}$ can be computed using the activation function Sigmoid (equation 1) to determine the final sum of weights.

$$
\mathrm{Y}(\mathrm{X})=\operatorname{Sig}[\mathrm{Z}(\mathrm{X})]
$$

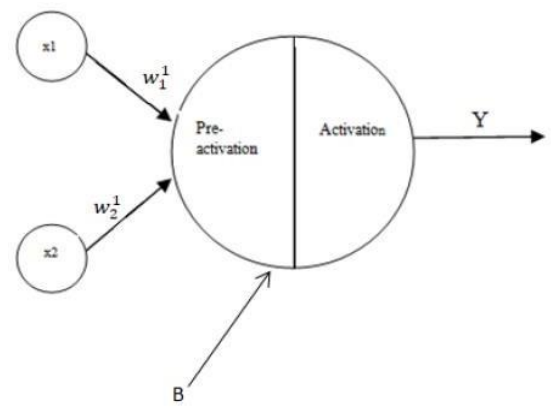

Figure 2. Pre-activation and activation phase within anode

Thus, a classification algorithm is used to classify an entry using the pre-trained learning model as follows.

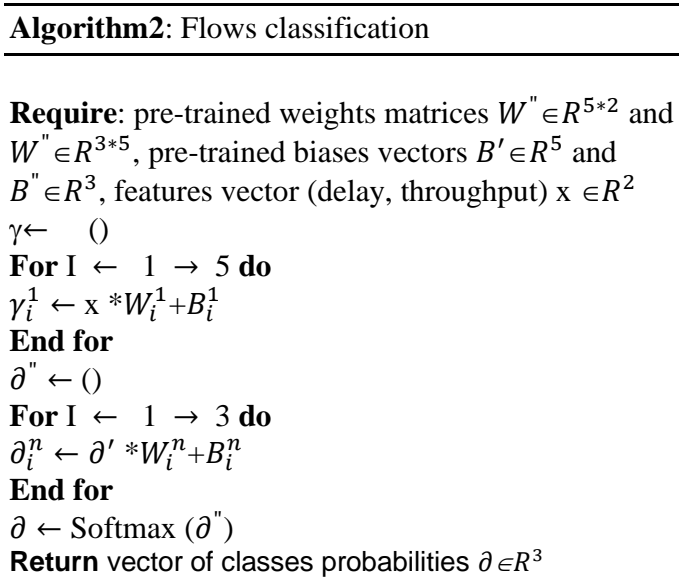

\subsection{Information Routing}

In SDN (Morzhov \& Nikitinskiy, 2018), coupling the control plane and the data plane has invented another method of routing packets. Routing becomes a communication between switches and SDN controllers instead of consulting a routing table in the routers. The classification method's mechanism is illustrated as follows: When the incoming traffic arrives at the switch, a "packet_In" is sent from the switch to the SDN controller indicating the characteristics of this stream. Since he has a general view of the network, the controller should calculate and make decisions (based on the proposed classification model). It then answers the switch with a 'paket_Out,' providing the decision for what host and how it will be directed. Assuming that this message is received, the switch can successfully orient the packet as the SDN controller indicates. 


\section{EVALUATION AND EXPERIMENTAL RESULTS}

In this section, we will describe the datasets selected for the experimental analyze and evaluate the proposed classification model's performance.

\subsection{Feature Extraction}

One of the most important steps in network traffic classification is the feature extraction as it is a crucial pre- processing task.

\subsubsection{Collect Data}

In reason of the lack of public and recent datasets available for research purposes, to achieve this step, forming an adequate dataset is mandatory. Traffic is captured during different periods with Wireshark. Obtained packets are saved into PCAP files. Initially, the program extracts the following features: duration for fixed packets, number of bytes, min packet length, max packet length, min interval packet time, max interval packet time and average interval packet time, the standard deviation of interval packet time, and the duration for fixed packets. Obtained features allow calculating the necessary inputs to our classification model: throughput and delay.

\subsubsection{Normalization}

Some inputs assume a finite number of values (discrete type) or continuous type. The data is normalized because it can have large order range values and different magnitudes, which can create problems in the classification. Thus, the values of the characteristics are normalized by applying a standard transformation to each characteristic. The normalization function is represented as follows.

$$
X=\left(x-x_{\min }\right)-\left(x_{\max }-x_{\min }\right)
$$

$x$ : The original value.

$x_{\min }$ : The minimum value of all attributes.

$x_{\text {max }}$ : The maximum value of all attributes.

$X$ : The normalized value $\in[0.1]$.

\subsubsection{Training and Test Data}

A large and representative set of data is one of the most important requirements for a deep learning model. The nodes of the $L_{i} ;$ i> 1 automatically choose and merge the information in the layer based on a weighted summation and an activation function. At the output layer, a measure of the average error is determined then used to adjust the weights throughout the network. This phase is named «Feed-forwarding» The final model is then used to predict the observations' responses in the second set of data called the validation dataset. We experiment with the proposed model on different sets of data. Each set will be automatically divided into $75 \%$ for the training and $25 \%$ for the validation.

\subsection{Performance}

In this section, we present the experimental results to evaluate our proposed classification model's performance in terms of precision.

\subsubsection{Settings of the Experiments}

To evaluate our model, the performance is measured in terms of precision. This metric is often defined as the proportion of the instances that correctly belong to class $X$ amidst all those classified as $X$. In this part, we evaluate our model with different epochs on the dataset generated during the phase of extraction of the characteristics: delay and throughput. The variation in precision is presented in figure 3 . 


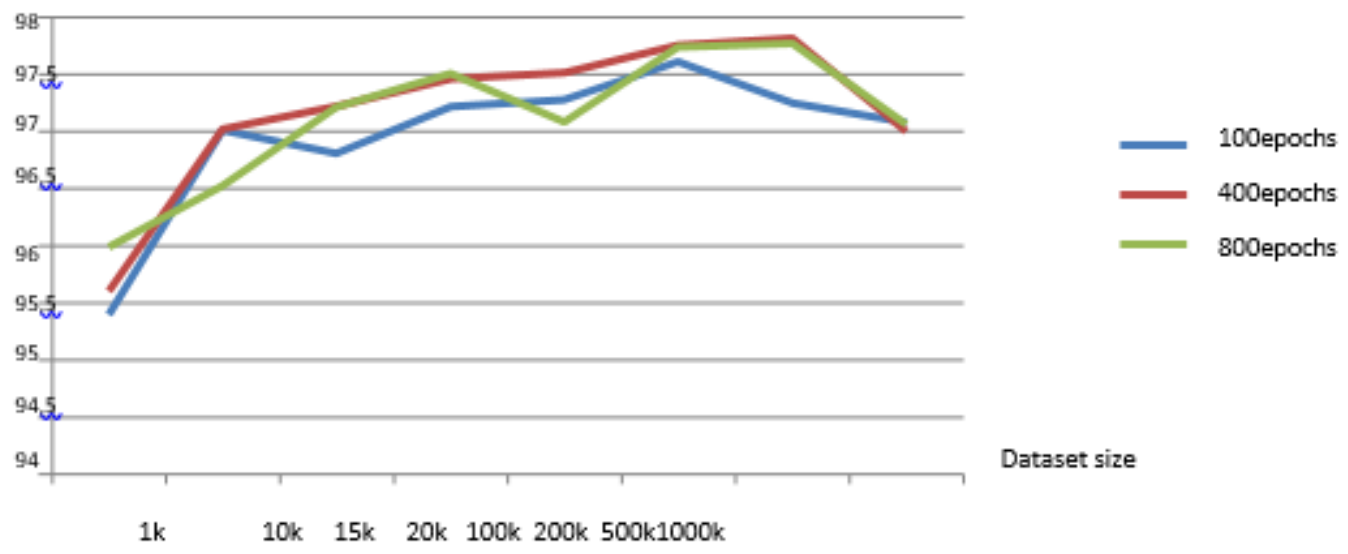

Figure 3. Precision of our model

Results shown in figure 3 present two phases: learning and over-learning. Testing data with a maximum number of flows 200k allows the model to learn continually, reaching a high accuracy of $97.74 \%$.The second phase starts with a dataset bigger than 200k. The precision curve decreases slightly but always has a significant value of precision equal to $96.99 \%$. As a result, the model achieved better than $97.5 \%$ Precision. This result allows the classifier to ensure an accurate classification over time.

Testing data in the flow interval] 200k, 500k [with 400 pockets allows the model to learn, achieving high accuracy of $97.8 \%$ gradually. The second phase begins when the number of incoming flows exceeds $500 \mathrm{k}$. The precision curve decreases slightly. It is a saturation phase i, e. A phenomenon appears when the weighted sum of the signals that most neurons receive as input is too large. A saturated network is often in a situation of over-learning. Therefore, we fixed the size of the learning bench at $200 \mathrm{k}$ in the rest of our experiment. This decision is made to finalize a compromise between execution time and accuracy. The results are illustrated in table 1 .

Table 1. Comparative study of execution time and precision between 200k and 500k models

\begin{tabular}{lll}
\hline & $200 \mathrm{k}$ & $500 \mathrm{k}$ \\
\hline Precision \% & 97.74 & 97.8 \\
\hline Execution time in sec & 24926.977 & 50188.597 \\
\hline
\end{tabular}

\subsubsection{Comparison}

To have a correct comparison, all the experiments are applied to the same dataset, including a standard performance measure. This section reviewed two supervised classification approaches, Logistic regression and Fuzzy decision tree, to accomplish this experience. The choice was based on the classification time, and accuracy. The indicators obtained in (Yan et al., 2015) reflect that the decision tree technique predicts students dropping out with more accuracy and a lower percentage of errors. In (Enajjari et al., 2010), it is shown that logistic regression takes a "Train Time" more than "Test Time," however, these two values are significantly minimized compared to other algorithms, namely the KNN. Therefore, these two algorithms have low complexity and show better results in various fields, namely prediction (Enajjari et al., 2010), and diagnostics (Sujatha et al., 2018).

\section{- The proposed model vs. Logistic regression (LR)}

Logistic regression (LR) is a mathematical model (Enajjari et al., 2010) with real observations. The principle of LR is to make optimization loops. To ameliorate LR's learning parameters and to find its higher precision value, we simulate it with a varied number of iterations. 


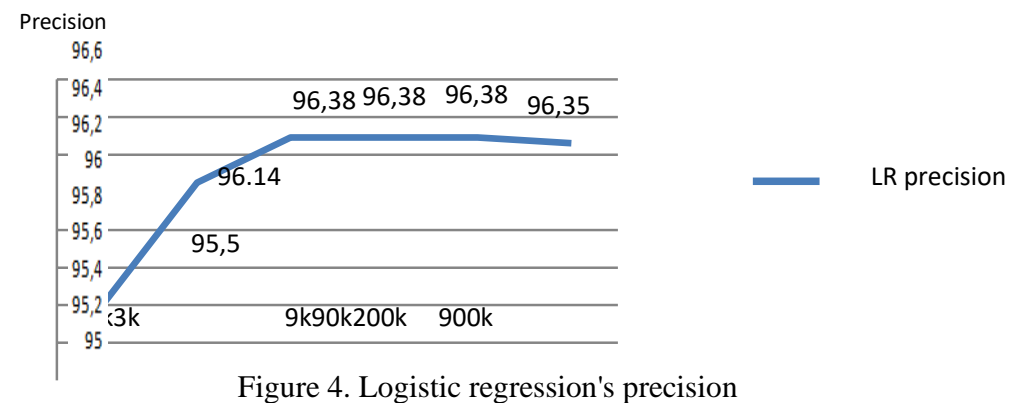

The highest precision obtained with LR is $96.38 \%$, which is lower than the proposed model's precision.

\section{- The proposed model vs. Fuzzy decision tree (FDT)}

The fuzzy decision tree is a sequential connected graph representing the succession of decisions (Sujatha et al., 2018). In (Olaru \& Wehenkel., 2003) is claimed that the performance of an FDT was based on the determination of its size. Thus, we try to build a tree, ensuring the best possible performance. Figure 5 shows the different results obtained. The decision tree presents a high accuracy, with $76.53 \%$.

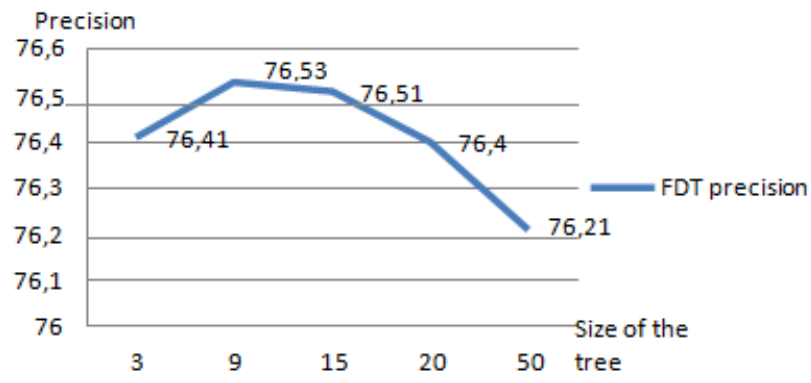

Figure 5. Precision of FDT

FDT is executed with its optimal depth (9 altitudes of nodes). We notice that the proposed model results are significantly better than FDT results in terms of precision.

\section{RESULTS}

We have shown that the proposed classification model based on Multilayer Perceptron is highly efficacious in precision, reaching approximately $97.74 \%$ with a high flows number reaching 500k flow, which presents a higher value compared to other solutions such as Fuzzy Decision Tree and Logistic regression.

\section{DISCUSSION AND FUTURE WORK}

In this paper, we have demonstrated an artificial neural network application to classify traffic by features. We investigate and propose an efficient model that can be deployed within SDN networks. It aims to classify flows into three classes: $\mathrm{AF}, \mathrm{EF}$, and $\mathrm{BE}$, based on delay and throughput. We illustrated the performance in terms of precision. The results indicate that our approach can achieve about $97.74 \%$ precision. Although the proposed method presents high precision performance, we must optimize it equally to its parameter execution time to start the system with a rapid time-based execution if applied to a real-time application.

\section{ACKNOWLEDGEMENT}

We want to thank everyone who helps to achieve this work. We made the used algorithms and the dataset openly accessible for the community working on this area. 


\section{REFERENCES}

Bar M., Ambaw i A. \& Doroslovacki M. (2018), "Comparison of Machine Learning Algorithms for Raw Handwritten Digits Recognition".

Burgstahler L., Dolzer K., Hauser C., Jahnert J., Junghans S., Macian C., \& Payer W. (2003), "Beyond technology: the missing pieces for QoS success", Association for Computing Machinery, New York, NY, USA, 121-130. DOI:https://doi.org/10.1145/944592.944597

Chan K., Babiarz J. \& Baker F. (2006), "Configuration guidelines for DiffServ service classes".

Da Silva A. S., Schaeffer-Filho A. E. \& Granville L. Z. (2015), "Identification and Selection of Flow Features for Accurate Traffic Classification in SDN".

En-Najjary, T. (2010). "Traffic classification: Application-based feature selection using logistic regression".

Hayes M., Ng B., Pekar A. \& Seah W. K. G. (2018), "Scalable Architecture for SDN Traffic Classification”.

Karagiannis T., Broido A., Brownlee N., Claffy K., \& Faloutsos M. (2004), “Is P2P dying or just hiding?" in IEEE Globecom.

Kogan K., Nikolenko S., Culhane W., Eugster P. \& Ruan E. (2013), "Towards efficient implementation of packet classifiers in SDN/OpenFlow," in Proc. 2nd ACM SIGCOMM Workshop Hot Topics Softw. Defined Netw.

Li Y. \& Li J. (2014), "MultiClassifier: A combination of DPI and ML for application-layer classification in SDN", The 2014 2nd International Conference on Systems and Informatics (ICSAI 2014), pp. 682-686.

Lin J. (2013), "Mapreduce is good enough? If all you have is a hammer, throw away everything that's not a nail!," Big Data, vol. 1, pp. 28-37.

Moore, A., \& Papagiannaki, K. (2005). "Toward the Accurate Identification of Network Applications". PAM.

Morzhov S. V. \& Nikitinskiy M. A. (2018), “Development\&research of the prerewall network application for oodlight SDN controller," in Proc. Moscow Workshop Electron. Netw. Technol. (MWENT), pp. 14.

Nandy M. (2013), "An Analytical Study of Supervised and Unsupervised Classification Methods for Breast Cancer Diagnosis", 2nd International conference on Computing Communication and Sensor Network (CCSN-2013).

Ng B., Winston M. H. \& Seah K.G. (2015), "Developing a Traffic Classification Platform for Enterprise Networks with SDN: Experiences and Lessons Learned", New Zealand.

Nguyen T. \& Armitage G. (2018) “A survey of techniques for internet traffic classification using machine learning," IEEE Communications Surveys Tutorials,Fourth.

Olaru C. \& Wehenkel L. (2003), “A complete fuzzy decision tree technique”, Fuzzy Sets\&Systems, Issue 2.

Pérez A., Grandón E., Caniupán M. \& Vargas G. (2019), "Comparative Analysis of Prediction Techniques to Determine Student Dropout: Logistic Regression vs Decision Trees".

Quoc D. L.,Alessandro V.D, Park B., Romano L. \& Fetzer C. (2015), "Scalable network traffic classification using distributed support vector machines," in Proc. IEEE 8th Int. Conf. Cloud Comput., New York, NY, USA.

Rezaei, S. \& Liu X. (2019), "Deep Learning for Encrypted Traffic Classification: An Overview”, Data Science and Artificial Intelligence for Communications.

Sandhya, S., Purkayastha, S., Joshua, E., \& Deep, A. (2017). Assessment of website security by penetration testing using Wireshark. 2017 4th International Conference on Advanced Computing and Communication Systems (ICACCS), 1-4.

Sen, S., Spatscheck, O., \& Wang, D. (2004). "Accurate, scalable in-network identification of p2p traffic using application signatures". WWW '04.

Sujatha R., AnithaNithya R., Subhapradha S., Srinithibharathi S. (2018), "Decision Tree Classification for Traffic Congestion",", International Journal of Engineering\&Techniques, India.

Yan J., Zhang H., Shuai Q., Liu B. \& Guo X. (2015), "HiQoS: An SDN-based multipath QoS solution", China Communications, 12(5): 123-133. 